Daimon. Revista Internacional de Filosofía, n ${ }^{\circ} 83,2021$ pp. 37-55

ISSN: 1130-0507 (papel) y 1989-4651 (electrónico)

http://dx.doi.org/10.6018/daimon.361811

Las obras se publican en la edición electrónica de la revista bajo una licencia Creative Commons ReconocimientoNoComercial-SinObraDerivada 3.0 España (texto legal). Se pueden copiar, usar, difundir, transmitir y exponer públicamente, siempre que: i) se cite la autoría y la fuente original de su publicación (revista, editorial y URL de la obra); ii) no se usen para fines comerciales; iii) se mencione la existencia y especificaciones de esta licencia de uso.

\title{
Las dimensiones éticas de los sistemas de valoración y difusión científica en el área de filosofía moral
}

\section{The ethical dimensions of systems of diffusion and scientific evaluation in the moral philosophy area}

\author{
RAMÓN A. FEENSTRA* \\ DANIEL PALLARÉS-DOMÍNGUEZ**
}

\begin{abstract}
Resumen: Los índices bibliométricos están adentrándose de manera progresiva en la realidad de la comunidad académica. Por un lado, conceptos como ranking de publicaciones y cuartiles se emplean en procesos de valoración como las acreditaciones, los sexenios o la concesión de proyectos. Por otro lado, las grandes editoriales están consolidando modelos de difusión de la información que dificultan su acceso para la comunidad investigadora. El objetivo de este artículo consiste en examinar críticamente la combinación que se produce entre la bibliometría, los sistemas imperantes de valoración de la investigación y los modelos editoriales. Este análisis nos lleva a esbozar algunos retos éticos que se plantea para el área de filosofía moral.

Palabras clave: ética, investigación, sistemas bibliométricos, modelos editoriales, acceso abierto.
\end{abstract}

\begin{abstract}
Bibliometric indices are progressively moving towards the reality of the academic community. On the one hand, concepts like rankings of publications and quartiles are used in evaluation processes, such as accreditations, sexennials or awarding projects. On the other hand, large publishing houses consolidate models to diffuse information which makes the access difficult for the research community. This article aims to critically examine the combination formed by bibliometry, prevailing systems that evaluate research and publishing models. This analysis allowed us to outline some ethical challenges that are considered for the moral philosophy area at different levels.
\end{abstract}

Keywords: ethics, research, bibliometric systems, publishing models, open access

Recibido: 05/02/2019. Aceptado: 28/03/2019.

* Profesor Titular de la Universitat Jaume I. feenstra@uji.es. Líneas de investigación: éticas aplicadas, ética de la comunicación, teoría de la democracia. Publicaciones: (2021), en coautoría con Emilio Delgado López-Cózar y Daniel Pallarés-Domínguez «Research misconduct in the fields of ethics and philosophy: researchers' perceptions in Spain, Science and Engineering Ethics, 27(1), 1-21. (2019). «La corregulación de la publicidad a debate», Revista Mediterránea de Comunicación. Núm. 2. Vol. 10, pp. 1-10.

** Profesor de filosofía en el IES Francisco Ribalta de Castellón y profesor asociado en la Universitat Jaume I de Castellón.dpallare@uji.es. Líneas de investigación: neuroética, neuroeducación, éticas aplicadas. Publicaciones: (2019), «Gobierno abierto: una concepción ética para la educación democrática», Revista del CLAD, $\mathrm{n}^{\circ} 73$. (2016), «Neuroeducación en diálogo. Neuromitos en el proceso de enseñanza-aprendizaje y en la educación moral», Pensamiento. Vol 72, n 273, pp. 941-958. 


\section{Introducción. Un modelo de tres piezas ${ }^{1}$}

La publicación de artículos de alto impacto se ha convertido en una actividad de extraordinaria importancia no solamente porque es un medio para divulgar el conocimiento, la reflexión crítica y los resultados de investigación sino también porque, a partir de ella, se aspira a progresar en la carrera académica. Conceptos complejos como ranking de publicaciones y cuartiles han irrumpido en nuestra realidad investigadora inmediata y se emplean en procesos de valoración como las acreditaciones, los sexenios o la concesión de proyectos. Por otro lado, las editoriales - sobre todo las grandes - están consolidando modelos de difusión de la información que basadas en suscripciones - de coste millonarios - dificulta, incluso para investigación financiada con dinero público, su acceso para la comunidad investigadora y todavía más para el público en general. La combinación entre los sistemas imperantes de valoración de la investigación, los modelos editoriales y la bibliometría marcan una serie de complejas reglas de juego no ajeno a numerosos debates de carácter ético. Este modelo afecta en la actualidad a todas las áreas de conocimiento y al área de filosofía moral con algunos matices significativos. Además, plantea una serie de retos tanto para investigadores, universidades, editoriales de carácter público e incluso para el Estado. El objetivo de este artículo consiste en examinar críticamente este modelo, así como reflexionar sobre las dimensiones micro, meso y macro a las que afecta y los dilemas que acompañan a cada nivel.

\section{Los métodos bibliométricos y de índices de impacto}

En las últimas décadas, las formas de evaluación y de difusión de la investigación ha venido acompañada de numerosos retos, tanto técnicos como éticos, que afectan a la totalidad del proceso editorial. En la diana de numerosas posiciones críticas del modelo actual aparece un punto clave de dicho paradigma: el Factor de Impacto - en adelante FI- (Hansson, 1995; Aleixandre-Benavent et al., 2004; De Vito, 2006; Moustafa, 2015; Reig, 2018). La incorporación de la bibliometría, como disciplina basada en la introducción de métodos matemáticos y estadísticos para medir la literatura científica, está suponiendo una transformación para todas las áreas de conocimiento, incluyendo las vinculadas con la filosofía y las humanidades tradicionalmente no acostumbradas a utilizar este tipo de métodos. La bibliometría se está aplicando con diferentes matices en función de las disciplinas pero, sin duda, parece que su herramienta más destacada, a la hora de realizar procesos de evaluación y valoración del avance científico, es lo que se conoce como el FI.

Este factor se extrae mediante el recuento del número de citas que anualmente obtiene una revista en relación a la cantidad de artículos publicados. El contar las referencias para clasificar el impacto de una revista fue introducido por Eugene Garfield (1955), aunque el

1 Este trabajo se inserta dentro de los objetivos del proyecto de la subvención para la contratación de personal investigador en fase postdoctoral (APOSTD/2017003) concedida por la Consellería de Educación, Cultura y Deporte de la Generalitat Valenciana así como dentro del proyecto de investigación, financiado por la Universitat Jaume I, "El potencial de las éticas aplicadas en las herramientas de participación del Gobierno Abierto y de la sociedad civil" (UJI-A2016-04). Los autores agradecen los consejos del profesor y doctor en Documentación Emilio Delgado López-Cózar 
término propiamente no fue usado hasta la publicación del Science Citation Index en 1963 (Moustafa, 2015). Si bien el Impact Factor publicado anualmente en los JCR - Journal Citation Reports, creado por el fenecido ISI, después Thomson Reuters, hoy en manos de Clarivate - fue el primer índice de impacto desarrollado, a este se le han sumado otros como los desarrollados por Elsevier-Scopus (Citescore, SJR y SNIP) así como también por Google Scholar (Índice h). Entre estos índices cabe destacar actualmente a WOS y Scopus como las dos principales bases de datos. Estos utilizan fórmulas mediante las cuales otorgan una posición determinada (cuartil) a una revista según el impacto - que se obtiene dividiendo el número de citas obtenidas por el número de textos publicados ${ }^{2}$. Con ello las revistas indexadas por estas bases de datos mejoran o empeoran cada año la posición dentro de su campo de conocimiento. El "valor" que estos factores de impacto conceden a la investigación, al menos desde el punto de vista numérico, se traslada del artículo a la revista ya que es esta la que obtiene una posición determinada cada año. Además, la mejoría (o empeoramiento) de una revista a lo largo de un año afecta en su conjunto a los autores que han publicado en ese canal de difusión - ya que el valor del "mérito" de una publicación se asigna a una cuestión numérica de citas obtenidas y no al propio contenido ${ }^{3}$. Todo ello presenta una notable incidencia sobre la proyección profesional de los investigadores quienes necesitan, cada vez más, sumar un número significativo de publicaciones con elevados impactos, tal y como observamos más adelante de forma detallada.

Actualmente los principales índices de impacto han pasado de ser una herramienta de medición de citas basado en una fórmula matemática, a ser indicadores supuestamente fiables para medir la calidad científica de las revistas. Es más, esta herramienta está convirtiéndose en el principal criterio para la contratación de científicos, la dotación de subvenciones y becas, la concesión de proyectos de investigación e incluso para medir la reputación científica de la comunidad investigadora (Campanario, 1999). El problema no es que los índices sean empleados para medir ciertos aspectos de la calidad científica, sino que se hayan convertido en unos instrumentos tan predominantes. Incluso el mismo creador del FI, Eugene Garfield, lo ha descartado para establecer un ranking cuando se habla de investigadores y personas (Correspondence Nature, 2003: 479). Las limitaciones de los índices de impacto como criterio autosuficiente son numerosas y entre éstas podrían destacarse los siguientes.

En primer lugar, el impacto se mide de forma arbitraria respecto a unos pocos años desde la publicación del artículo. Para disciplinas del campo de las humanidades y la filosofía en particular esto supone un perjuicio frente a disciplinas de carácter empírico más acostumbradas a citar los "últimos avances" (Hansson, 1995; Seglen, 1997; De Vito, 2006; Moustafa, 2015).

2 Por ejemplo, CiteScore de Scopus extrae el factor de impacto dividiendo las citas obtenidas durante un año específico por el número de artículos publicados en los tres últimos años. Para el recuento sólo se contemplan las citas que adquiere los artículos publicados durante ese mismo periodo de 3 años. Ejemplo de la fórmula. CiteScore $=$ Citas obtenidas en el año 2019/documentos 2016-2018. A partir del factor CiteScore se calcula los índices SJR (Scimago) así como SNIP. Esta información puede consultarse en: https://www.scopus.com

3 El 12 de junio de 2018 el Tribunal Supremo dio la razón al recurso interpuesto por la profesora Amparo Sánchez Segura obligando a la CNEAI a revisar y leer el contenido de los trabajos presentados en el sexenio. Con ello se lograba una sentencia histórica que resolvía contra la tendencia de denegar sexenios basándose exclusivamente en el impacto de las revistas. 
En segundo lugar, los índices de impacto no discriminan las razones por las que se cita un artículo y estas pueden ser muy variadas, incluyendo las que aluden a la mala calidad o pobreza científica de los trabajos. La reproducibilidad científica también se ve afectada en este sentido. Lo que más preocupa a los grupos de investigación son los hot research topics antes que en verificar o intentar reproducir lo que otros han publicado. El actual sistema de indexación fuerza a las revistas a publicar lo que se prevé que se va a citar más en el futuro, para que así suba en los índices de impacto (Moustafa, 2015: 141; D'Antonio Maceiras, 2018).

En tercer lugar, los índices de impacto no tienen en cuenta las condiciones sociales de cada país ni la totalidad de las diferencias y matices que existen entre áreas de investigación dentro del ámbito nacional, favoreciendo las investigaciones y publicaciones del ámbito angloamericano (Aleixandre-Benavent et al., 2004).

\section{La evaluación de la investigación en el área de filosofía moral}

Si bien el uso de la bibliometría, basada en medir la investigación en función del factor de impacto que se obtiene de acuerdo al número de citas obtenidas, lleva aplicándose un par de décadas en el contexto español (Orduña-Malea, Martín-Martín y Delgado, 2017), para el área de filosofía moral y áreas afines su uso es mucho más reciente. Además, su uso no es idéntico entre los diferentes procesos de valoración, ya que varía en los procesos de valoración de sexenios y de acreditaciones a los cuerpos de titular o catedrático.

De esta manera, la Comisión Nacional Evaluadora de la Actividad Investigadora (CNEAI), en el campo 11 "Filosofía, Filología y Lingüística" señala de manera textual que:

se tendrá en cuenta como referencia de calidad la inclusión en bases de datos internacionales como el «Arts and Humanities Citation Index» de la «Web of Science», «Journal Citation Reports, Social Sciences Edition», Emerging Sources Citation Index y Scimago Journal Rank ${ }^{4}$.

Así pues, la CNEAI considera como puntos de referencia de calidad, utilizando la terminología de la FECYT, a "las dos principales bases de datos mundiales de referencias bibliográficas y citas de publicaciones periódicas" Web of Science (WoS), propiedad de Clarivate Analytics, y Scopus, propiedad de Elsevier ${ }^{5}$. De la convocatoria de la CNEAI cabe destacar que también considera información recogida en otras bases de datos, indicadores y sistemas de clasificación de revistas como Ulrichsweb, CAPES, ERIH Plus, MIAR, inRECH (en los años disponibles), RESH y CAHRUS. Como último elemento destacable de esta convocatoria llama la atención que la CNEAI, a pesar de apuntar la importancia de que la

4 Esta convocatoria puede ser consultada en el siguiente enlace: https:/www.boe.es/boe/dias/2018/11/26/pdfs/ BOE-A-2018-16138.pdf

5 La descripción e información que proporciona la FECYT sobre bases de datos resulta de interés para conocer estas bases de datos. Enlace disponible en https://www.recursoscientificos.fecyt.es/

Las guías de la BUS que proporcionan la Bliobioteca de la Universidad de Sevilla son también de gran utilidad para la academia: http://guiasbus.us.es/c.php?g=129439\&p=845019 
publicación en revista "deberá de ser al menos de nivel medio", no utiliza el lenguaje de cuartiles a la hora de valorar los sexenios en la rama de filosofía.

Por su parte, la Agencia Nacional de Evaluación de la Calidad y Acreditación (ANECA) incluye una mayor cantidad de herramientas bibliométricas en sus procesos de valoración de la investigación para las figuras de titular y catedrático. De esta manera, para la comisión de Ciencias Sociales (D18) - donde se incluye el área de filosofía moral ${ }^{6}$ - se exige, para lograr una valoración A de investigación, "aportar un mínimo de 50 publicaciones, de las cuales, al menos, 30 deberán ser artículos de revistas" para la figura de catedrático/a, y "al menos, 30 publicaciones, de ellas 20 como mínimo serán artículos de revistas" para la figura de titular. Además, la convocatoria específica que, para el primero de los casos se precisará de "20 artículos de nivel 1" mientras que para el caso de titular se reclaman "10 publicaciones de los niveles 1 y 2 " 7 . La ANECA introduce de pleno el lenguaje los factores de impacto y cuartiles ya que los niveles 1, 2 y 3 de revistas se fijan en función del cuartil que ocupa la publicación, produciéndose ligeras variaciones entre las figuras de catedrático/a y de titular.

\begin{tabular}{|l|l|l|l|}
\hline $\begin{array}{c}\text { Categorías según } \\
\text { ANECA }\end{array}$ & Índice JCR (WOK) & $\begin{array}{c}\text { Índice SJR } \\
\text { (Scopus) }\end{array}$ & \multicolumn{1}{|c|}{ Otros índices } \\
\hline Nivel 1 & $\begin{array}{l}\text { Revistas en el Q1 o } \\
\text { Q2 }\end{array}$ & Q1 & \\
\hline Nivel 2 & Q3 & Q2 & \\
\hline Nivel 3 & Q4 & Q3 y Q4 & $\begin{array}{l}\text { Sello de calidad de la FECYT o } \\
\text { indexado en ESCI }\end{array}$ \\
\hline
\end{tabular}

Tabla 1. Niveles de revistas para la figura de Catedrático/a área Ciencias Sociales.

\begin{tabular}{|l|l|l|l|}
\hline $\begin{array}{c}\text { Categorías según } \\
\text { ANECA }\end{array}$ & Índice JCR (WOK) & $\begin{array}{c}\text { Índice SJR } \\
\text { (Scopus) }\end{array}$ & \multicolumn{1}{|c|}{ Otros índices } \\
\hline Nivel 1 & $\begin{array}{l}\text { Revistas en el Q1, Q2 } \\
\text { o Q3 }\end{array}$ & Q1 y Q2 & \\
\hline Nivel 2 & Revistas en Q4 & Q3 & $\begin{array}{l}\text { Sello de calidad de la FECYT o } \\
\text { indexado en ESCI }\end{array}$ \\
\hline Nivel 3 & & Q4 & \\
\hline
\end{tabular}

Tabla 2. Niveles de revistas para la figura de Titular área Ciencias Sociales.

La introducción de la bibliometría y los factores de impacto como mecanismo principal de valoración de la investigación tiene numerosas consecuencias sobre el desarrollo profesional de las personas vinculadas a la universidad (Rodríguez-Serrano y Gil Solvedilla,

6 El área D18 de Ciencias Sociales se compone por: Antropología Social; Biblioteconomía y Documentación, Ciencia Política y de la Administración; Comunicación Audiovisual y Publicidad; Filosofía Moral; Historia del Pensamiento y de los Movimientos Sociales y Políticos; Periodismo; Sociología; Trabajo Social y Servicios Sociales.

7 Convocatoria disponible en: http://www.aneca.es/Programas-de-evaluacion/Evaluacion-de-profesorado/ACADEMIA/Criterios-de-evaluacion-noviembre-2017 
2018). Antes de adentrarnos en algunas de estas es relevante atender otro punto que marca el desarrollo de la investigación: los modelos editoriales.

\section{Los modelos editoriales de las grandes corporaciones: financiación y difusión de la información}

Otra cuestión clave del modelo actual es la financiación y modelo de negocio de las revistas de investigación y especialmente de aquellas pertenecientes a las cinco grandes editoriales, Reed-Elsevier, Taylor \& Francis, Wiley-Blackwell, Springer y Sage, que son precisamente las que copan los cuartiles superiores de los índices de impacto (D'Antonio Maceiras, 2018). El ecosistema de revistas es complejo y plural, pero hay una serie de rasgos que se han consolidado en torno a la forma de producir y consolidar publicaciones periódicas.

Uno de ellos es su dependencia respecto al conjunto de la comunidad investigadora. Así, por ejemplo, en los procesos de revisión son los investigadores los que ejercen de evaluadores de la calidad de los trabajos. La colaboración del conjunto de la comunidad científica es una pieza clave del proceso. Los revisores otorgan un enorme valor a la investigación, comentan, discuten, contribuyen a su mejora e incluso a su selección. De los procesos de revisión depende que la revista seleccione buenos trabajos y el logro de una publicación concreta no es ajena a esta labor, casi siempre altruista, que desarrollan la comunidad investigadora. Paralelamente, se produce una circunstancia particular y es que muchos de los investigadores (aunque no todos) proceden de universidades que se financian con dinero público. El valor que aporta la comunidad investigadora al resultado final de las publicaciones de todas las ramas parece fuera de duda siendo esta la que asume, por un lado, las labores de producción con las autorías y, por otro lado, las tareas de control de calidad en tanto que editores y revisores.

Por otra parte, a esta estructura cabe sumar otro elemento y es que las editoriales como modelo de negocio obtienen sus beneficios mediante políticas muy concretas basadas en el acceso a la investigación (Buranyi, 2017). Las grandes editoriales dan acceso a los resultados de investigación, por lo general, mediante el pago de suscripciones. Además, lo hacen mediante la venta de paquetes de revistas en un contexto en el cual se consolidan tendencias de carácter oligopolístico ya que, por introducir un par de datos solamente, las 10 editoriales más grandes constituyen el $45 \%$ de las revistas indexadas en Scopus y, todavía más relevante, concentran el 75,5\% de las revistas de mayor impacto. Es decir, son las que copan los primeros cuartiles de los rankings bibliométricos que, a su vez, son las revistas donde necesita publicar la comunidad investigadora si quiere promocionar (Colledge et al., 2010: 218).

Las editoriales que amasan un conjunto amplio de revistas de prestigio y alto impacto promocionan luego el acceso a las revistas mediante contratos elevados que suscriben por cantidades millonarias con los Estados y las universidades. Sin ir más lejos, y a pesar de la poca transparencia en los costes de suscripción a revistas científicas, se ha dado a conocer que España paga anualmente a Elsevier unos 25 millones de euros, mientras que Finlandia asume unos costes de unos 9 millones y Alemania hasta el 2018 - año en el que renuncia a mantener las suscripciones, tal y como veremos más adelante - de unos 10 millones (Villarreal y Escudero, 16 febrero 2018). Este modelo de negocio se ha visto ampliado de manera 
que, no solo logran que instituciones públicas hagan frente al pago de caras suscripciones, sino que en algunos casos - haciendo uso del prestigio de muchas publicaciones así como de la necesidad imperante del investigador por publicar - se incorpora la práctica de solicitar a los investigadores el cobro por publicar8.

Evidentemente, no todas las editoriales y publicaciones tienen el mismo modelo y hay algunas que apuestan por modelos de financiación diferentes, pero hoy en día las cifras indican que solamente el $25 \%$ de las investigaciones se publican con acceso abierto ${ }^{9}$ (Science Metrix, 2018; Open access directory, 2018). Y esto se hace por diferentes motivos, como el apoyo financiero por parte de las universidades (Research information network, 2009), el tipo de licencia de creative commons por el que se opta (IMPublicationsOpen, 2018), o incluso en algunos campos por la finalidad estratégica de compartir información de forma más abierta entre diferentes grupos de información (Spires-Jones et al., 2016).

La relación entre la distribución de trabajo y esfuerzos de revisión, el acceso de la información y la financiación de las editoriales ha llevado a significativos debates y cuestionamientos sobre el modelo actual. Y no es para menos dado que estamos ante un sistema donde es el Estado el que financia gran parte de la investigación mediante proyectos, el que se hace cargo de la remuneración de una parte importante de los investigadores - quienes revisan la calidad de la investigación - y luego el que compra la mayor parte del trabajo que ha sido publicado. Es lo que se conoce como un sistema de triple pago (Buranyi, 2017).

\section{Algunas consecuencias de este modelo}

Las consecuencias que derivan de esta combinación entre sistemas imperantes de valoración de la investigación, los modelos editoriales y la bibliometría afectan a diversos niveles: 1) individual como investigadores, 2) institucional como universidades o editoriales de revistas y 3) estatal, especialmente por lo que afecta a la financiación y acceso.

En el plano individual no es extraño que los sistemas de valoración de la investigación hayan fomentado en la comunidad investigadora una preocupación constante por publicar, un fenómeno que se ha venido a conocer popularmente como publish or perish. La promoción e incluso permanencia en el ámbito académico de un investigador depende en gran parte de su capacidad de publicar una prolífica cantidad de trabajos y sobre todo de publicar en los formatos a los que se concede valía y reconocimiento - revistas de "alto impacto". Los investigadores se ven en la coyuntura de seleccionar con acierto no solo las temáticas que van a trabajar y desarrollar sino también dónde aspiran a publicarlas. Para el investigador las revistas que no están en lo alto de los índices resultan, al menos en términos estratégicos, menos atractivas y es que, el "éxito" bibliométrico de las publicaciones marcan las posibilidades de éxito en acreditaciones, promociones, sexenios, etc. Las áreas de filosofía moral y filosofía se enfrentan a la dificultad añadida de que incluso las revistas españolas con "mayor impacto" en índices no llegan a alcanzar la valoración de nivel 1

8 Según autores como Castejón (2018) y Aréchaga (2011b), se calcula que los precios aproximados para un artículo de diez páginas en blanco y negro puede llegar a alcanzar los 1500 euros, 2000 si es a color, e incluso en algunos casos pueden llegar a los 5000 dependiendo del FI que tenga esa revista.

9 Es interesante consultar el Directory of Open Access Repositories (OpenDOAR) para ver la progresiva evolución del Open Access. Ver: http://v2.sherpa.ac.uk/opendoar/ 
que exige la ANECA para la acreditación a titular o catedrático (Ver tabla 3). Aspecto que puede fomentar el interés por publicar especialmente en revistas — en su práctica totalidad revistas del ámbito anglosajón- pertenecientes a las grandes editoriales y ubicadas en las partes superiores de los cuartiles. La comunidad científica se ve enfrascada en una cultura muy competitiva y no es extraño que ello pueda fomentar cierto incremento de las malas prácticas en la investigación científica, tales como el plagio, la falsificación o manipulación de la información, etc., (Helgesson \& Eriksson, 2014; Hvistendahl, 2013).

\begin{tabular}{|c|c|c|c|c|c|c|c|}
\hline $\begin{array}{l}\text { Título de } \\
\text { Revista }\end{array}$ & \begin{tabular}{|l|} 
Índice H \\
(Google \\
Scholar)
\end{tabular} & $\begin{array}{l}\text { Sello } \\
\text { calidad } \\
\text { FECYT } \\
2014 / 2016\end{array}$ & $\begin{array}{l}\text { SJR } \\
2017\end{array}$ & $\begin{array}{l}\text { Arts and } \\
\text { Humanities } \\
\text { (Clarivate) }\end{array}$ & \begin{tabular}{|l|} 
Emerging \\
Sources \\
(Clarivate)
\end{tabular} & $\begin{array}{l}\text { Ranking } \\
\text { Redib 2017 }\end{array}$ & \begin{tabular}{|l|} 
ICDS \\
MIAR \\
$2017^{10}$
\end{tabular} \\
\hline Agora & $\begin{array}{l}\mathrm{H} \text { index: } 4 \\
\text { Mediana H: } 5\end{array}$ & $\checkmark$ & - & - & $\checkmark$ & \begin{tabular}{l|}
76.716 \\
$6 / 37$ filosofía \\
$242 / 748$ \\
general \\
\end{tabular} & 10.0 \\
\hline $\begin{array}{l}\text { Anuario } \\
\text { Filosófico }\end{array}$ & $\begin{array}{l}\text { H index: } 4 \\
\text { Mediana H: } 5\end{array}$ & $\checkmark$ & $\begin{array}{l}0,1 \\
\mathrm{Q} 4\end{array}$ & $\checkmark$ & $\checkmark$ & Sí & 11.0 \\
\hline Araucaria & \begin{tabular}{|l|} 
H index: 6 \\
Mediana H: 14
\end{tabular} & $\checkmark$ & $\begin{array}{l}0,12 \\
\text { Q3 }\end{array}$ & - & $\checkmark$ & $\begin{array}{l}48.627 \\
18 / 37 \\
\text { filosofía } \\
512 / 748 \\
\text { general } \\
\end{array}$ & 9.8 \\
\hline Astrolabio & $\begin{array}{l}\text { H index: } 3 \\
\text { Mediana H: } 4\end{array}$ & - & - & - & - & - & 3.6 \\
\hline Bajo Palabra & $\begin{array}{l}\mathrm{H} \text { index: } 4 \\
\text { Mediana H: } 6\end{array}$ & - & - & - & $\checkmark$ & $\begin{array}{l}52.746 \\
475 / 748 \\
\text { general }\end{array}$ & 7.8 \\
\hline Comprendre & - & $\checkmark$ & $\begin{array}{l}\text { Indexada } \\
\text { sin factor } \\
\text { de impacto } \\
2017\end{array}$ & - & - & & 9.8 \\
\hline Constelaciones & $\begin{array}{l}\text { H index: } 4 \\
\text { Mediana H: } 9\end{array}$ & - & - & - & $\checkmark$ & \begin{tabular}{l|}
74.815 \\
$8 / 37$ filosofía \\
$266 / 748$ \\
general \\
\end{tabular} & 4.4 \\
\hline $\begin{array}{l}\text { Con-Textos } \\
\text { Kantianos } \\
\text { (CTK) }\end{array}$ & $\begin{array}{l}\text { H index: } 4 \\
\text { Mediana H: } 9\end{array}$ & - & $\begin{array}{l}0,11 \\
\text { Q3 }\end{array}$ & - & $\checkmark$ & $\begin{array}{l}41.341 \\
25 / 37 \\
\text { filosofía } \\
584 / 748 \\
\text { general }\end{array}$ & 9.0 \\
\hline Contrastes & $\begin{array}{l}\text { H index : } 4 \\
\text { Mediana H: } 5\end{array}$ & $\checkmark$ & - & - & - & - & 6.5 \\
\hline
\end{tabular}

10 La tabla no incluye el FI de Clarivate debido a que ninguna de las revistas españolas de filosofía y ética está recogida por este índice de impacto. Cabe recordar brevemente que la sección de Arts y Humanities de Clarivate sí incluye una serie de estas revistas pero que no cuenta con FI. 


\begin{tabular}{|c|c|c|c|c|c|c|c|}
\hline Convivium & - & - & $\begin{array}{l}0,1 \\
\text { Q4 }\end{array}$ & $\checkmark$ & - & $\begin{array}{l}58.669 \\
14 / 37 \\
\text { filosofía } \\
416 / 748 \\
\text { general }\end{array}$ & 10.0 \\
\hline $\begin{array}{l}\text { Cuadernos de } \\
\text { bioética }\end{array}$ & $\begin{array}{l}\text { H index: } 7 \\
\text { Mediana H: } 17\end{array}$ & - & $\begin{array}{l}0,11 \\
\text { Q4 } \\
\text { (medicina) }\end{array}$ & - & $\checkmark$ & \begin{tabular}{|l|}
66.262 \\
$2 / 2$ ética \\
$278 / 748$ \\
general
\end{tabular} & 9.9 \\
\hline $\begin{array}{l}\text { Cuadernos } \\
\text { Salmantinos de } \\
\text { Filosofía }\end{array}$ & $\begin{array}{l}\text { H index: } 3 \\
\text { Mediana H: } 4\end{array}$ & - & \begin{tabular}{|l|} 
Indexada \\
sin factor \\
de impacto \\
2017 \\
\end{tabular} & - & - & - & 6.5 \\
\hline Daimon & $\begin{array}{l}\text { H index: } 8 \\
\text { Mediana H: } 10\end{array}$ & $\checkmark$ & $\begin{array}{l}0,13 \\
\text { Q3 }\end{array}$ & - & $\checkmark$ & $\begin{array}{l}54.013 \\
17 / 37 \\
\text { filosofía } \\
464 / 748 \\
\text { general }\end{array}$ & 10.0 \\
\hline Debats & $\begin{array}{l}\text { H index: } 3 \\
\text { Mediana H: } 3\end{array}$ & - & - & - & $\checkmark$ & - & 10.0 \\
\hline $\begin{array}{l}\text { Diálogo } \\
\text { Filosófico }\end{array}$ & - & - & - & - & - & - & 4.5 \\
\hline Dilemata & $\begin{array}{l}\text { H index: } 9 \\
\text { Mediana H: } 11\end{array}$ & - & - & - & - & - & 3.9 \\
\hline Eikasia & $\begin{array}{l}\text { H index: } 5 \\
\text { Mediana H: } 5\end{array}$ & - & - & - & $\checkmark$ & - & 4.6 \\
\hline Endoxa & $\begin{array}{l}\text { H index: } 5 \\
\text { Mediana H: } 7\end{array}$ & $\checkmark$ & $\begin{array}{l}0,11 \\
\text { Q3 }\end{array}$ & - & $\checkmark$ & \begin{tabular}{|l|}
40.813 \\
$24 / 37$ \\
filosofía \\
$582 / 748$ \\
general \\
\end{tabular} & 9.9 \\
\hline Enrahonar & $\begin{array}{l}\text { H index: } 3 \\
\text { Mediana } \mathrm{H}: 3\end{array}$ & $\checkmark$ & - & & $\checkmark$ & \begin{tabular}{|l|}
31.204 \\
$27 / 37$ \\
filosofía \\
$663 / 748$ \\
general \\
\end{tabular} & 10.0 \\
\hline $\begin{array}{l}\text { Estudios } \\
\text { Filosóficos } \\
\end{array}$ & $\begin{array}{l}\text { H index: } 3 \\
\text { Mediana H: } 4\end{array}$ & - & - & - & - & - & 6,5 \\
\hline Isegoría & $\begin{array}{l}\text { H index: } 7 \\
\text { Mediana H: } 14\end{array}$ & $\checkmark$ & $\begin{array}{l}0,15 \\
\text { Q3 }\end{array}$ & $\checkmark$ & & \begin{tabular}{|l|}
80.306 \\
$5 / 37$ filosofía \\
$227 / 748$ \\
general \\
\end{tabular} & 10.0 \\
\hline Pensamiento & $\begin{array}{l}\text { H index: } 4 \\
\text { Mediana } \mathrm{H}: 7\end{array}$ & - & $\begin{array}{l}0,19 \\
\text { Q2 }\end{array}$ & $\checkmark$ & & - & 11.0 \\
\hline $\begin{array}{l}\text { Ramon Llull } \\
\text { Journal of } \\
\text { Applied Ethics }\end{array}$ & - & - & $\begin{array}{l}0,1 \\
\text { Q4 }\end{array}$ & - & - & - & 9,3 \\
\hline Recerca & $\begin{array}{l}\text { H index: } 11 \\
\text { Mediana H: } 16\end{array}$ & $\checkmark$ & $\begin{array}{l}0,11 \\
\text { Q3 }\end{array}$ & - & $\checkmark$ & \begin{tabular}{|l|}
89.388 \\
$3 / 37$ filosofía \\
$154 / 748$ \\
general
\end{tabular} & 10.0 \\
\hline
\end{tabular}




\begin{tabular}{|c|c|c|c|c|c|c|c|}
\hline $\begin{array}{l}\text { Revista de } \\
\text { Filosofía } \\
\text { (UCM) }\end{array}$ & $\begin{array}{l}\text { H index: } 8 \\
\text { Mediana H: } 5\end{array}$ & $\checkmark$ & $\begin{array}{l}0,1 \\
\text { Q4 }\end{array}$ & - & $\checkmark$ & \begin{tabular}{|l|}
66.367 \\
$9 / 37$ filosofía \\
$344 / 748$ \\
general \\
\end{tabular} & 10.0 \\
\hline $\begin{array}{l}\text { Revista de } \\
\text { Estudios } \\
\text { Orteguianos }\end{array}$ & $\begin{array}{l}\text { H index: } 2 \\
\text { Mediana H: } 3\end{array}$ & - & $\begin{array}{l}0,1 \\
\mathrm{Q} 4\end{array}$ & - & $\checkmark$ & - & 7.7 \\
\hline $\begin{array}{l}\text { Revista de } \\
\text { Hispanismo } \\
\text { Filosófico }\end{array}$ & $\begin{array}{l}\text { H index: } 2 \\
\text { Mediana H: } 3\end{array}$ & - & $\begin{array}{l}0,1 \\
\text { Q4 }\end{array}$ & $\checkmark$ & - & - & 8.9 \\
\hline Teorema & $\begin{array}{l}\text { H index: } 8 \\
\text { Mediana H: } 10 \\
\end{array}$ & $\checkmark$ & \begin{tabular}{|l}
0,11 \\
$\mathrm{Q} 3$
\end{tabular} & $\checkmark$ & - & & 11.0 \\
\hline Theoria & $\begin{array}{l}\mathrm{H} \text { index: } 8 \\
\text { Mediana H: } 10\end{array}$ & $\checkmark$ & $\begin{array}{l}0,14 \\
\text { Q3 }\end{array}$ & $\checkmark$ & - & & 11.0 \\
\hline Thémata & $\begin{array}{l}\text { H index: } 4 \\
\text { Mediana H: } 7\end{array}$ & - & - & - & $\checkmark$ & \begin{tabular}{|l}
45.987 \\
$21 / 37$ \\
filosofía \\
$539 / 748$ \\
general \\
\end{tabular} & 8.0 \\
\hline $\begin{array}{l}\text { Torres de } \\
\text { Lucca }\end{array}$ & $\begin{array}{l}\text { H index: } 3 \\
\text { Mediana H: } 6\end{array}$ & - & $\begin{array}{l}\text { Indexada } \\
\text { sin factor } \\
\text { de impacto }\end{array}$ & - & $\checkmark$ & $\begin{array}{l}44.615 \\
22 / 37 \\
\text { filosofía } \\
548 / 748 \\
\text { general }\end{array}$ & 7.2 \\
\hline
\end{tabular}

Tabla 3. Revistas españolas de filosofía y/o ética. Elaboración propia ${ }^{11}$.

En el plano institucional este modelo provoca una serie de consecuencias tanto para las universidades como para las editoriales. Las universidades se enfrentan a un escenario donde la distribución de la financiación o su posicionamiento en diversas clasificaciones de universidades a lo largo del mundo (el de Taiwán o el de Shanghái, por mencionar dos ejemplos) se vinculan también al éxito de su profesorado en los índices bibliométricos. Por su parte, el campo de las revistas es evidentemente otra afectada. Para las revistas españolas del área de filosofía moral y áreas cercanas se da la peculiaridad de que son en su gran mayoría de acceso abierto y vinculadas a universidades o centros de investigación públicos (Tabla 4). Con la adopción progresiva de la lógica bibliométrica por parte de investigadores, universidades y revistas, las publicaciones se ven inmersas en una compleja espiral. Las revistas que salen "peor paradas" en la "foto" de la bibliometría tienen una mayor dificultad de recibir un número significativo de textos y pueden verse empujadas a "luchar" por ascender su posición - primero por lograr ser indexadas en las grandes bases de datos Scopus y Clarivate, más adelante por escalar posición dentro de los cuartiles. Las revistas que logran ascender en este terreno complejo se ven, sin embargo, en una situación

11 Esta tabla ha sido elaborada utilizando las siguientes fuentes y bases de datos: Scimago Journal Rank (SJR): https://www.scimagojr.com/; Matriz de Información para el Análisis de Revistas (MIAR): http://miar.ub.edu/; Sello de Calidad de la FECYT: http://www.evaluacionarce.fecyt.es/Publico/index.aspx; Google Scholar Metrics:https://scholar.google.com/citations?view_op=metrics_intro\&hl=en; Ranking de revistas REDIB: https://www.redib.org/recursos/Ranking/Revistas. Asimismo, para los datos de index $\mathrm{H}$ se ha seguido la investigación de Delgado López-Cózar y Martín-Martín (2018). 
particular y es que su éxito bibliométrico se puede erigir a su vez en una amenaza para su sostenibilidad. La subida en los factores de impacto de una publicación se ve acompañada por una exponencial multiplicación de las propuestas que recibe. Para las revistas privadas de las grandes editoriales, altamente profesionalizadas y con grandes presupuestos anuales para la gestión, la edición y la maquetación, esto no supone un grave problema, pero es diferente para revistas vinculadas a instituciones públicas donde los presupuestos son más limitados y donde el trabajo - también editorial - es desarrollado por lo general por académicos que desarrollan su labor de manera combinada con otras obligaciones académicas. En un contexto globalizado y ampliamente competitivo las revistas se ven forzadas a desarrollar modelos editoriales sostenibles y muchas veces con recursos escasos.

\begin{tabular}{|c|c|c|}
\hline Título de Revista & Institución & Enlace \\
\hline Agora & Universidade de Santiago de Compostela & http://www.usc.es/revistas/index.php/agora \\
\hline Anuario Filosófico & Universidad de Navarra & $\begin{array}{l}\text { https://www.unav.edu/publicaciones/revistas/ } \\
\text { index.php/anuario-filosofico/index }\end{array}$ \\
\hline Araucaria & Universidad de Sevilla & http://institucional.us.es/araucaria/ \\
\hline Astrolabio & Universitat de Barcelona & https://www.raco.cat/index.php/Astrolabio \\
\hline Bajo Palabra & Universidad Autónoma de Madrid & https://revistas.ucm.es/index.php/RESF \\
\hline Comprendre & $\begin{array}{l}\text { Universitat Ramon Llull y editorial } \\
\text { Herder }\end{array}$ & https://www.herdereditorial.com/comprendre \\
\hline Constelaciones & $\begin{array}{l}\text { Centro de Ciencias Humanas y Sociales } \\
\text { del CSIC }\end{array}$ & http://constelaciones-rtc.net/ \\
\hline $\begin{array}{l}\text { Con-Textos } \\
\text { Kantianos (CTK) }\end{array}$ & $\begin{array}{l}\text { Consejo Superior de Investigaciones } \\
\text { Científicas, CSIC: Instituto de Filosofía } \\
\end{array}$ & $\begin{array}{l}\text { https://www.con-textoskantianos.net/index. } \\
\text { php/revista/index }\end{array}$ \\
\hline Contrastes & Universidad de Málaga & $\begin{array}{l}\text { http://www.revistas.uma.es/index.php/ } \\
\text { contrastes }\end{array}$ \\
\hline Convivium & Universitat de Barcelona & https://www.raco.cat/index.php/Convivium \\
\hline $\begin{array}{l}\text { Cuadernos de } \\
\text { bioética }\end{array}$ & $\begin{array}{l}\text { Asociación Española de Bioética y Ética } \\
\text { Médica }\end{array}$ & $\begin{array}{l}\text { http://aebioetica.org/cuadernos-de-bioetica. } \\
\text { html }\end{array}$ \\
\hline $\begin{array}{l}\text { Cuadernos } \\
\text { Salmantinos de } \\
\text { Filosofía }\end{array}$ & Universidad Pontificia de Salamanca & $\begin{array}{l}\text { https://www.upsa.es/facultades/detalle-facultad/ } \\
\text { contenido.php?idCtro=2513\&idCG=3404 }\end{array}$ \\
\hline Daimon & Universidad de Murcia & https://revistas.um.es/daimon \\
\hline Debats & $\begin{array}{l}\text { Institució Alfons el Magnànim-Centre } \\
\text { Valencià d'Estudis i de Recerca } \\
\end{array}$ & $\begin{array}{l}\text { http://www.revistadebats.net/index. } \\
\text { php?journal=debats }\end{array}$ \\
\hline Diálogo Filosófico & Universidad Pontificia de Salamanca & http://www.dialogofilosofico.com/ \\
\hline Dilemata & Independiente & $\begin{array}{l}\text { https://www.dilemata.net/revista/index.php/ } \\
\text { dilemata }\end{array}$ \\
\hline Eikasia & Eikasia Ediciones & http://www.revistadefilosofia.org/ \\
\hline Endoxa & $\begin{array}{l}\text { Universidad Nacional de Educación a } \\
\text { Distancia (UNED) }\end{array}$ & http://revistas.uned.es/index.php/endoxa \\
\hline Enrahonar & Universitat Autònoma de Barcelona & https://revistes.uab.cat/enrahonar \\
\hline Estudios Filosóficos & $\begin{array}{l}\text { Instituto Superior de Filosofía de } \\
\text { Valladolid de la Orden de Predicadores }\end{array}$ & http://estudiosfilosoficos.dominicos.org/ \\
\hline
\end{tabular}




\begin{tabular}{|l|l|l|}
\hline Título de Revista & Institución & Enlace \\
\hline Isegoría & $\begin{array}{l}\text { Consejo Superior de Investigaciones } \\
\text { Científicas, CSIC: Instituto de Filosofía }\end{array}$ & $\begin{array}{l}\text { http://isegoria.revistas.csic.es/index.php/ } \\
\text { isegoria }\end{array}$ \\
\hline Pensamiento & Universidad Pontificia Comillas & hensamiento \\
\hline $\begin{array}{l}\text { Ramon Lull Journal } \\
\text { of Aplied Ethics }\end{array}$ & $\begin{array}{l}\text { Universitat Ramon Llull y editorial } \\
\text { Herder }\end{array}$ & https://www.raco.cat/index.php/rljae \\
\hline Recerca & Universitat Jaume I de Castelló & http://www.e-revistes.uji.es/index.php/recerca \\
\hline Revista de Filosofía & Universidad Complutense de Madrid & https://revistas.ucm.es/index.php/RESF \\
\hline $\begin{array}{l}\text { Revista de Estudios } \\
\text { Orteguianos }\end{array}$ & Fundación José Ortega y Gasset & $\begin{array}{l}\text { https://ortegaygasset.edu/publicaciones/revista- } \\
\text { de-estudios-orteguianos/ }\end{array}$ \\
\hline $\begin{array}{l}\text { Revista de } \\
\text { Hispanismo } \\
\text { Filosófico }\end{array}$ & Asociación de Hispanismo Filosófico,, & http://ahf-filosofia.es/?page_id=33 \\
\hline Teorema & KRK Ediciones & $\begin{array}{l}\text { https://www.unioviedo.es/Teorema/Spanish/ } \\
\text { index.html }\end{array}$ \\
\hline Theoria & Universidad Pais Vasco & http://www.ehu.eus/ojs/index.php/THEORIA \\
\hline Thémata & Universidad de Sevilla & http://www.lastorresdelucca.org/index.php/ojs \\
\hline Torres de Lucca & Universidad Complutense de Madrid & histes/es/revistas/themata- \\
\hline
\end{tabular}

Tabla 4. Relación entre las revistas españolas y la institución de la que dependen. Elaboración propia

En el plano estatal las consecuencias ya han sido explicitadas anteriormente y se refieren a la financiación de todo el entramado científico. Los respectivos gobiernos se enfrentan a una situación donde no solo hacen posible las investigaciones académicas sino que además luego deben pagar por resultados que se han generado con dinero público. Una situación que al menos parece peculiar. De hecho, el modelo que aquí venimos describiendo ha llevado a que a lo largo de los últimos años se multipliquen, como se verá a continuación, las voces críticas y los modelos alternativos, donde la ética es un factor clave de la gobernanza de la investigación científica (López de la Vieja, 2016).

\section{Respuestas por parte de la comunidad investigadora y de los Estados}

Las respuestas críticas frente al modelo actual son numerosas y de carácter heterogéneo. Sin pretender hacer una revisión a todas estas, sí resulta de interés introducir algunas significativas que se podrían agrupar en: modelos revisionistas, rupturistas y revolucionarios. Estas posiciones tienen en común su crítica frente al modelo existente y su defensa favorable a introducir cambios en las políticas de difusión y valoración de la investigación. Les separa, sin embargo, el grado de ruptura que promocionan con respecto al modelo editorial, al acceso abierto y al uso de los índices de impacto. Cada uno de estos modelos se centra en una de las dos cuestiones que podríamos denominar clave, y que conforman el ethos científico actual: la representatividad y calidad - representado por los índices de impacto- y la transparencia - representada por el acceso abierto. 
El modelo revisionista se caracteriza por la intención de mejorar el proceso editorial, en favor de los investigadores y restando poder a los grandes conglomerados empresariales en una línea continuista, pero a la vez crítica con el sistema (D’Antonio Maceiras, 2018: 470). Las iniciativas revisionistas no intentan romper con el modelo establecido, pero sí eliminar ciertas asperezas que condicionan las carreras investigadoras. La mayoría de estas iniciativas se centran en los índices de impacto. Como ejemplo principal del modelo revisionista podemos citar la Declaration on Research Assessment (DORA).

DORA, firmada en San Francisco en 2012 por cientos de investigadores e instituciones académicas constituye un ejemplo paradigmático de intento por reformar el sistema. Esta declaración aboga principalmente por eliminar el FI en la evaluación de los individuos, pero no de los trabajos científicos. Propone una evaluación sometida a una pluralidad de factores y actores, basándose en la teoría de los stakeholders. Es decir, el modelo revisionista de la DORA pretende que el FI no sea determinado únicamente por la mecánica actual de dividir el número de citas entre la cantidad de artículos que una revista publica en un plazo determinado (Medawar, 1964; Crane 1967). Tres son las recomendaciones principales que realiza: 1) eliminar el uso de las métricas basadas en revistas como baremo principal y medida sustantiva en todas aquellas acciones que tengan que ver con financiación, contratación, promoción de personas - seguirá estando como parte del baremo, pero de forma secundaria; 2) evaluar la investigación por sus méritos propios, en función de la calidad del artículo y no de la calidad de la revista; y 3) aprovechar y favorecer la publicación en abierto y todos los recursos de publicación online.

Por su parte, entendemos por modelo rupturista toda iniciativa que pretende transformar el modelo editorial de publicación científica actual, pero sin atacar directamente a los grandes conglomerados empresariales anteriores, y por supuesto sin romper el marco legal. Los casos que se pueden incluir dentro de esta postura se caracterizan por intentar renegociar condiciones - cuotas de pago, precios de suscripciones - con los grandes conglomerados empresariales y romper aquellos acuerdos que se consideran abusivos. Dos son los ejemplos paradigmáticos: el cOAlition $S$ plan, y las rupturas de países como Alemania y Francia con los gigantes editoriales. Si las iniciativas revisionistas anteriores se centraban especialmente en los índices de impacto, los modelos rupturistas se centran en el acceso abierto.

Por un lado, el cOAlition $S$ plan (Science Europe, 2018), que tiene como principal objetivo la implantación del acceso abierto de forma inmediata en todas las publicaciones e investigaciones realizadas con fondos públicos. En efecto, el open Access haría la totalidad del proceso de publicación más accesible, eficiente, justo, dotándolo de mecanismos de transparencia y monitorización. En apoyo a iniciativas como la mencionada anteriormente, DORA, el cOAlition $S$ plan pretende instaurarse en enero de 2020. Entre las recomendaciones que realiza a autores, editores, plataformas, revistas y repositorios, se pueden destacar:

1) La utilización del DOI como identificador permanente de un artículo, y reunir los metadatos en revistas de alto nivel -incluyendo las referencias bibliográficas - bajo un formato interoperable estándar y de dominio público.

2) El completo y permanente acceso de los autores a sus propias investigaciones sin ninguna restricción, haciendo uso del Creative Common License y en colaboración con DOAJ -Directory of Open Access Journals. 
3) Hacer un reparto justo y equitativo de los costes de publicación, donde se puedan proporcionar ayudas económicas que favorezcan a los autores.

A pesar de que el cOAlition $S$ plan pueda mostrar una línea propositiva, lo cierto es que rompe directamente con el núcleo central del negocio científico con las grandes empresas. El acceso abierto supondría un verdadero y necesario proceso de democratización de la ciencia.

Por otro lado, dentro de las tendencias rupturistas se cuentan con ejemplos de países como Alemania o Francia que han puesto fin a los acuerdos editoriales con empresas como Springer-Nature o Elsevier. El consorcio francés Couperin.org rompió en 2018 su acuerdo con Springer-Nature. Couperin representa a 250 universidades y a centros como el Centre National de la Recherche Scientifique, y ha cancelado el contrato con la editorial y las 2000 revistas a las que tiene suscripción, debido a la subida de precios (Villareal \& Escudero, 18 de abril 2018). También Alemania se ha sumado a la iniciativa de no renovar contratos con grandes editoriales. La biblioteca digital de la Max Planck Society (MPS) no reanudará las suscripciones de Elsevier, a partir de su finalización en diciembre de 2018.

Finalmente, podemos diferenciar una serie de movimientos que, a nuestro juicio, se pueden definir como revolucionarios en la medida que actúan fuera del marco legal y buscan provocar un cambio cualitativo del sistema de difusión de la investigación. Estas iniciativas entienden que el modelo editorial y de publicación actual encabezado por las grandes compañías empresariales se caracteriza por una concentración excesiva de poder y por el aprovechamiento de los fondos públicos.

El principal ejemplo lo constituye, por ser el de mayor envergadura y alcance, $\mathrm{Sci}-\mathrm{Hub}$, creado por Alexandra Elbakian en 2011. Lo que pretendía esta investigadora de posgrado de informática de Kazajistán, era crear un sitio web donde la mayoría de las investigaciones del mundo estuvieran disponibles de forma gratuita para cualquier persona que tuviera acceso a la red. En 2016, Elsevier emprendió acciones legales contra Elbakian, quien a día de hoy se ha visto condenada a pagar 15 millones de euros de indemnización al gigante editorial. Considerada por algunos como la Robin Hood de la ciencia académica (Aluffi, 9 de febrero 2017) ha puesto 62 millones de artículos en formato de acceso abierto y gratuito para los investigadores en la conocida Science-Hub. A pesar de haberse convertido en enemiga pública de las grandes editoriales, Elbakian ha sido incluso nominada por Nature como una de las 10 personas más influyentes de la historia y difusión de la ciencia (Ferrer, 17 de junio de 2016). Elbakian es considerada como la sucesora de Aaron Swartz, quien ayudó a crear la tecnología RSS, y cofundó Reddit y Open Library. Después de descargar más de 20 millones de páginas de Spacer, y pretender hacer lo mismo con JSTOR después, se vio enfrascado en un proceso judicial mediante el cual se le demandó con una multa millonaria. Swatrz terminó suicidándose pero su gesto quedó como el primer gran acto de desobediencia frente al modelo editorial (Gil, 17 enero de 2013). 


\section{Conclusiones}

El modelo imperante de valoración y difusión de la investigación es, tal y como se ha tratado de esbozar en estas páginas, altamente complejo y los retos que genera son abundantes. A pesar de que las respuestas y resistencias por parte de la sociedad académica y civil, así como también de diversos organismos estatales, van en aumento, las soluciones a los desequilibrios, los costes y los problemas que genera no parecen sencillas (Maceiras, 2018). Dentro de la propia comunidad científica están aumentando las voces y los análisis críticos. Paradójicamente, los estudios procedentes del área de filosofía moral sobre la ética de la investigación no ocupan en el momento actual el lugar central que seguramente merece por su trascendencia y actualidad. El área de filosofía moral puede, sin embargo, suponer un campo clave desde el cual generar futuros trabajos de investigación y de transferencia al ámbito de la ética de la investigación. La revisión que aquí se ha planteado a un modelo de tres piezas - la bibliometría, la evaluación de la investigación y los modelos editoriales - , remiten precisamente a campos centrales de las éticas aplicadas y muy especialmente a la ética del consumo (Cortina, 2002; Michelletti, 2010), la ética empresarial o la ética de las instituciones y de las políticas públicas (Calvo 2014; González Esteban, 2007; García Marzá, 2004; Cortina, 1993). Junto a otros trabajos de carácter sociológico, sería de enorme interés que la comunidad académica vinculada al área de filosofía moral ahondará en las herramientas o prácticas necesarias para abordar un escenario donde se produce una evidente colonización de las humanidades por parte de los métodos cuantitativos (Coin, 2019). El trabajo que aquí se presenta representa solo un paso inicial con el que se busca enmarcar ciertos retos éticos a los que nos vemos abocados.

La ética del consumo es un terreno propicio para examinar las exigencias normativas que se derivan de nuestro uso de las fuentes académicas. Son numerosas las preguntas que surgen en el desarrollo de una trayectoria investigadora ¿deberíamos tratar de publicar en revistas de acceso abierto y darle prioridad frente a otros modelos de difusión ${ }^{12}$ ? ¿Es justo realizar (de forma gratuita) trabajos de revisión de artículos de publicaciones que exigen elevadísimos costes de acceso a la comunidad científica? ¿En qué medida puedo como investigador transformar el sistema imperante si luego las agencias de valoración priorizan las revistas con "alto impacto”? ¿Es importante apoyar las revistas de instituciones públicas para que crezcan en un ambiente competitivo o desigual o es mejor no entrar en el juego bibliométrico y esperar a cambiar el sistema en su conjunto?

La ética aplicada también es un terreno, que desde el nivel meso (García-Marzá, 2016), nos empuja a reconsiderar las responsabilidades que tienen las instituciones tanto a nivel de revistas como de universidades ¿deberían las revistas de instituciones públicas tratar de competir en este sistema, mantenerse como están o quizás debería renunciar en su empeño y "fusionarse" a las grandes editoriales? O con respecto a las grandes editoriales privadas ¿podemos exigirles más desde el punto de vista ético o es aceptable que usen su posición privilegiada? Por lo que respecta a las universidades o centros de investigación ¿deberían utilizar con más frecuencia otros sistemas de valoración y medición de la investigación y

12 Recientemente Delgado López-Cózar (2018) ha propuesto una serie de buenas prácticas útiles para investigadores, gestores y bibliotecarios con el título de "La hoja de ruta verde de la comunicación científica". 
no dejarlo en manos principalmente de las grandes bases de datos? ¿Cuáles podrían ser los sistemas alternativos? ¿Qué herramientas institucionales debería o podría incorporar cada institución de cara a asesorar a sus investigadores frente a los dilemas morales (además de jurídicos) que acompañan a sus publicaciones? El incorporar la perspectiva ética y las dimensiones individual e institucional que acompañan a este paradigma puede resultar un campo relevante en un futuro cercano tanto en el desarrollo de trabajos críticos como de ética aplicada.

Finalmente, también es relevante resaltar el papel que tienen las agencias de evaluación estatales y los propios Estados en el modelo de investigación actual. Las voces críticas van en aumento con posturas heterogéneas - revisionistas, rupturistas o revolucionarias - y estas coinciden en resaltar la responsabilidad que tienen también el Estado. Para áreas como la de filosofía moral se produce incluso la paradoja de que se exigen prácticamente "imposibles" dentro de la carrera docente y la acreditación a titular y catedrático cuando se solicita un número significativo de publicaciones en revistas de los primeros cuartiles - la base de datos como Arts y Humanities (WOS) no otorga cuartiles a las revistas y en SJR las revistas indexadas en español de filosofía moral no están en los cuartiles superiores. Las dificultades del modelo actual de investigación, muchas veces achacadas en exclusiva a los índices de impacto, son fruto también del uso que las instituciones estatales y también universitarias hacen de estos índices. La adecuación a las especificidades de cada área, así como la elaboración de formas alternativas de evaluación parecen una tarea urgente en un futuro inmediato.

\section{Bibliografía}

Aleixandre-Benavent, Rafael, González de Dios, Javier, Valderrama-Zuriñan, Juan Carlos y Granda-Orive, José Ignacio (2004), «El factor de impacto. Un polémico indicador de calidad científica», Revista española de economía de la salud, Vol. $3, \mathrm{n}^{\circ} 5$.

Aluffi, Giuliano (9 de febrero de 2017), «Es cierto: robo a los editores para dárselo a los científicos». El País. Disponible en: https://elpais.com/elpais/2017/02/06/ciencia/1486399819_243966.html. Consultado el 30 de diciembre de 2018.

Aréchaga, Juan (15 de septiembre de 2011a), «Los españoles y las revistas científicas... ¡Que editen ellos!». El País. Disponible en: https://elpais.com/sociedad/2011/09/15/ actualidad/1316037621_850215.html. Consultado el 10 de marzo de 2019.

Aréchaga, Juan (2011b), «El prestigio y la rentabilidad de las revistas científicas españolas se basa en el uso internacional de sus contenidos», Endocrinología y nutrición, vol. 58, $\mathrm{n}^{\mathrm{o}} 2$, pp. 57-61.

Buranyi, Stephen (2017), «iSon los vertiginosos beneficios de la industria editorial malos para la ciencia?». Sinpermiso. Disponible en: http://www.sinpermiso.info/textos/sonlos-vertiginosos-beneficios-de-la-industria-editorial-malos-para-la-ciencia (Consultado el 2 de enero 2019).

Calvo, Patrici (2014), «Ética empresarial, responsabilidad social y bienes comunicativos», Tópicos. Revista de Filosofía, no 47, pp. 199-232.

Campanario, Juan Miguel (1999), «La ciencia que no enseñamos», Enseñanza de las ciencias, vol. 17, n 3 , pp. 397-410. 
Castejón, Francisco (3 de octubre de 2018), «El oscuro negocio de las revistas académicas». Ctxt revista contexto. Disponible en: https:/ctxt.es/es/20181003/Culturas/22049/ Francisco-Castejon-academicos-articulos-monopolio-alcance.htm. Consultado el 10 de marzo de 2019.

Coin, Francesca (2019), «La ineptitud del digital academic: precariedad y salud en el mundo universitario», Recerca. Revista de Pensament i Análisi, nº 24, 114-133.

Colledge, Lisa; Moya-Anegon, Felix; Guerrero-Bote, Vicente y Moek, Henk (2010), «SJR and SNIP: two new journal metrics in Elsevier's Scopus», Serials, vol. 23, no 3, pp. 215-221.

Correspondence Nature (2003), «Challenging the tyranny of the impact factors», Nature, $\mathrm{n}^{\mathrm{o}}$ 423, pp. 479-480.

Cortina, Adela (1993), Ética aplicada y democracia radical, Madrid: Tecnos.

Cortina, Adela (2002), Por una ética del consumo, Madrid: Taurus.

Crane, Diana (1967), «The gatekeepers of science: Some factors affecting the selection of articles for scientific journals», The American Sociologist, vol. 2, no 4, pp. 195-201.

D’Antonio Maceiras, Sergio (2018), «El circulo vicioso de las revistas científicas y la progresiva irrelevancia de la ciencia pública», Política y Sociedad, vol. 55, n 2, pp. 467-490.

De Vito, Eduardo (2006), «Algunas consideraciones en torno al uso del Factor de Impacto en la Bibliometría como herramienta de evaluación científica», Revista Americana de Medicina Respiratoria, vol. 6, $\mathrm{n}^{\mathrm{o}}$ 1, pp. 37-45.

Delgado López-Cózar, Emilio (2018), «De la ruta de oro a la ruta verde de la comunicación científica: negocio editorial y bibliométrico, publicación libre, acceso abierto, evaluación total e individualizada», Revistas Científicas de Educación en Red. Pre-print, pp. 1-9.

Delgado López-Cózar, Emilio y Martín-Martín, Alberto (2018), «Índice H de las revistas científicas españolas según Google Scholar Metrics (2013-2017). 2a ed», Granada, 18 diciembre 2018.

DORA (2012), Declaration on research assessment. San Francisco: DORA.

Ferrer, Sergio (17 de junio de 2016), «La joven que desafía a las editoriales con su 'Pirate Bay’ de artículos científicos». El confidencial. Disponible en: https://www.elconfidencial. com/tecnologia/2016-06-17/scihub-alexandra-elbakyan-snowden-swartz_1218538/. Consultado el 30 de diciembre de 2018.

García-Marzá, Domingo (2004), Ética empresarial: del diálogo a la confianza, Madrid: Trotta.

García-Marzá, Domingo (2016), «Enfoques mesodeliberativos: sobre la articulación institucional en las democracias deliberativas», ISEGORÍA. Revista de filosofía moral y política, $\mathrm{n}^{\circ}$ 54, pp. 147-170.

Garfield, Eugene (1955), «Citation indexes for science: a new dimension in documentation through association of ideas», Science, $\mathrm{n}^{\circ} 122$, pp. 108-111.

Gil, Ivan (17 de enero de 2013), «¿Depresión o conspiración? El enigma de Aaron Swartz». El confidencial. Disponible en: https:/www.elconfidencial.com/almacorazon-vida/2013-01-17/depresion-o-conspiracion-el-enigma-de-aaron-swartz_204746 Consultado el 11 de marzo de 2019. 
González Esteban, Elsa (2007), «La teoría de los “stakeholders”: un puente para el desarrollo práctico de la ética empresarial y de la responsabilidad social corporativa», VERITAS: Revista de Filosofía y Teología, ${ }^{\circ}$ 17, pp. 205-224.

Hane, Paula (2002), «The Prestige (Factor) is Gone: This start-up competitor to ISI's Journal Impact Factor has recently been forced out of business», Information Today, vol. 19, $\mathrm{n}^{\circ} 5$.

Hansson, Sture (1995), «Impact factor as a misleading tool in evaluation of medical journals», Lancet, $\mathrm{n}^{\circ} 346$, pp. 906.

Helgesson, Gert y Eriksson, Stefan (2014), «Plagiarism in research. Medicine Health Care and Philosophy», doi: 10.1007/s11019-014-9583-8.

Hvistendahl, Mara (2013), «China’s publication Bazaar», Science, vol. 342, n 6162, pp. 1035-1039.

IMPublicationsOpen (2018), Plan S. Disponible en: https://www.impopen.com/blog/plan-s. Consultado el 3 de enero de 2019.

López de la Vieja, Teresa (2016), «Ethics and governance in translational research», Ethics, Medicine and Public Health, $\mathrm{n}^{\circ} 2$, pp. 256-262.

Medawar, Peter B. (1964), «Is The Scientific Paper a Fraud? Experiment: A Series of Scientific Case», Experiment: A Series of Scientific Case Histories First Broadcast in the BBC Third Programme, British Broadcasting Corporation, London, pp. 7-13.

Michelletti, Michele (ed.) (2010), Political Virtue and Shopping. Individuals, Consumerism, and Collective Action. Nueva York: Palgrave.

Moustafa, Khaled (2015), «The Disaster of the Impact Factor», Science and Engineering Ethics, $\mathrm{n}^{\circ} 21$, pp. 139-142.

Open Access Directory (2018), OA by the numbers. Disponible en: http://oad.simmons.edu/ oadwiki/OA_by_the_numbers. Consultado el 3 de enero de 2019.

Orduna-Malea, Enrique, Martín-Martín, Alberto y Delgado López-Cózar, Emilio (2017), «Métricas en perfiles académicos: ¿un nuevo juego adictivo para los investigadores?», Revista Española de Salud Pública, vol. 90, 22 de septiembre, e1-e5.

Projekt Deal (2017), Projekt Deal. Bundesweite Lizenzierung von Angeboten großer Wissenschaftsverlage. Disponible en: https://www.projekt-deal.de/about-deal/. Consultado el 19 de diciembre de 2018.

Reig, Ramón (2018), «La paciencia, madrasta de la ciencia», en: Rodríguez-Serrano, Aarón y Gil-Solevilla, Samuel (eds.), Investigar en la era neoliberal, Barcelona: Aldea Global, pp. 155-186.

Research information Network (2009), Guidance for higher education and research institutions, publishers and authors, London: Universities UK and Research information network.

Rodríguez-Serrano, Aarón y Gil-Soldevilla, Samuel (eds.) (2018), Investigar en la era neoliberal, Barcelona: Aldea Global.

Science Europe (2018), cOAlition S. Making full and immediate Open Access a reality. Disponible en: www.coalition-s.org. Bélgica: Science Europe. Consultado el 27 de diciembre de 2018.

Science Metrix (2018), Analytical Support for Bibliometrics Indicators. Open Access availability of scientific publications, Montreal: Science Metrix Inc. 
Seglen, Per O. (1997), «Why the impact factor of journals should not be used for evaluating research». BMJ, $\mathrm{n}^{\circ} 314$, pp. 498-502.

Spires-Jones, Tara; Poirazi, Panayiota y Grubb, Mathew S. (2016), «Opening up: open access publishing, data sharing, and how they can influence your neuroscience carrer», European Journal of Neuroscience, vol. 43, pp. 1413-1419.

The Retraction Watch (2018), «Retraction watch. Tracking retractions as a window into the scientific process». Disponible en: http://retractionwatch.com/. Consultado el 5 de enero de 2019.

Villarreal, Antonio y Escudero, Jesús (18 de abril de 2018), «Francia rompe con la editora de 'Nature' mientras España aún suelta una millonada», El confidencial. Disponible en: https://www.elconfidencial.com/tecnologia/ciencia/2018-04-18/francia-rompe-springernature-millones-euros_1551443/. Consultado el 4 de enero de 2019.

Villarreal, Antonio y Escudero, Jesús (16 de febrero de 2018), «Esto es lo que cuestan las revistas científicas: España se ha pulido 213 millones desde 2012». El confidencial. Disponible en: https://www.elconfidencial.com/tecnologia/ciencia/2018-02-16/revistascientificas-suscripciones-millonada_1522586. Consultado el 10 de marzo de 2019.

Villarreal, Antonio y Escudero, Jesús (21 de febrero de 2018), «Elsevier paralizó una ley clave para proteger su millonario monopolio en España». El confidencial. Disponible en: https://www.elconfidencial.com/tecnologia/ciencia/2018-02-21/editoriales-elsevieropen-access-desactiva_1524848/. Consultado el 9 de marzo de 2019. 